\title{
Comparación de la normalización lineal de la escala de tiempos con el registro funcional continuo en movimientos cíclicos del cuello
}

\author{
López-Pérez, Noelia ${ }^{a}$; Venegas, William ${ }^{\text {; }}$ Serra-Añó, Pilarc; Page, Álvaro ${ }^{\text {d }}$ \\ ${ }^{a}$ E.T.S. I. Industriales. Universitat Politècnica de València (España); noelope1@etsii.upv.es. \\ bEscuela Politécnica Nacional de Quito (Ecuador); William.venegas@epn.edu.ec \\ 'Facultat de Fisioterapia, Universitat de València (España); pilar.serra@uv.es \\ dnstituto Universitario Mixto de Biomecánica, Universitat Politècnica de Valencia (España); \\ afpage@ibv.upv.es
}

\begin{abstract}
The time scale normalization is a necessary step in the analysis of human movements. The standard technique is linear normalization that may be ineffective in reducing the variability in the duration of events. An alternative is the registration of functions that involves adjusting the time scale in a nonlinear way, a complex and computationally expensive procedure.

In this work, both methods are compared for the cyclic movement of flexionextension of the neck. From a database with 437 complete cycles, we have analyzed the differences in mean curves and functional standard deviations, whose mean values and confidence intervals have been established through a bootstrapping process.
\end{abstract}

The results show small, although significant, differences in the average curves obtained, with a greater amplitude in the angles and velocities. The functional standard deviations are somewhat lower when registration is used. These results suggest that the continuous registration, although it may be useful for the analysis of non-periodic signals, offers no significant advantages over linear normalization in the case of cyclic movements.

Keywords: Human movement analysis, linear normalization of the time scale, Registration of functions; Functional Data Analysis.

\section{Resumen}

La normalización de la escala de tiempos es un paso necesario en el análisis de movimientos humanos. La técnica estándar es la normalización lineal que puede ser ineficaz para reducir la variabilidad en la duración de los eventos. Una alternativa es el registro continuo que supone ajustar de forma no lineal la escala de tiempo, procedimiento complejo y computacionalmente costoso. 
Comparación de la normalización lineal de la escala de tiempos con el registro funcional continuo en movimientos cíclicos del cuello

En este trabajo se comparan ambos métodos para el movimiento cíclico de flexo-extensión del cuello. A partir de una base de datos con 437 ciclos completos, se han analizado las diferencias en las curvas medias y las desviaciones típicas funcionales, cuyos valores medios e intervalos de confianza se han establecido mediante un proceso de bootstrapping.

Los resultados muestran pequeñas, aunque significativas, diferencias en las curvas medias obtenidas, con una mayor amplitud en los ángulos y velocidades. Las desviaciones típicas funcionales son algo menores en las curvas registradas. Estos resultados sugieren que el registro no lineal, aunque puede ser útil para el análisis de señales no periódicas, no ofrece ventajas importantes frente a la normalización lineal en el caso de los movimientos cíclicos.

Palabras clave: Análisis de movimientos humanos, Normalización lineal de la escala de tiempos, Registro de funciones; Análisis de Datos Funcionales.

\section{Introducción}

Los estudios clásicos sobre Biomecánica describen el movimientos humanos mediante variables numéricas tales como máximos, mínimos, rangos de movimiento, velocidades y aceleraciones máximas, duración de fases del movimiento, etc. (Winter, 1991; Ciriello et al, 1991; Novacheck, 1998). Este planteamiento supone reducir la información contenida en las funciones continuas que representan todas las variables cinemáticas o dinámicas y limitar la posibilidad de analizar las relaciones existentes entre las variables y sus derivadas.

A partir del año 2000 surge un cambio en la Estadística clásica motivado por la publicación de varios trabajos sobre una nueva forma de abordar el estudio de señales continuas, denominada Análisis de Datos Funcionales (DFA) (Ramsay \& Silverman, 2005). El DFA plantea una alternativa en la que se considera el carácter continuo de las variables de movimiento y permite desarrollar técnicas de tratamiento adaptadas a ese tipo de datos. La idea básica del Análisis de Datos Funcionales (FDA) reside en la descripción de cada observación como una función continua dependiente del tiempo (Lucero, 2000; Mechmeche, et al, 2016). Los métodos de FDA son capaces de extraer información, contenida en las señales o funciones y sus derivadas, que no se encuentra normalmente aplicando métodos de la estadística tradicional (Ullah \& Finch, 2013).

Sin embargo, para que las curvas de diferentes pruebas o sujetos sean comparables, es necesario ajustar la base de tiempos de alguna manera, de forma que se separe la variabilidad asociada a la amplitud de la relacionada con diferencias en el patrón temporal 
(Liebl \& Mosler, 2012). En efecto, las diferencias de fase introducen mucha variabilidad en la muestra de curvas, lo que hace muy difícil su comparación directa y determina que los valores medios no representen una buena estimación del conjunto (Sørensen \& Sangalli, 2013; Crane et al., 2011). Es en estos casos en los que se debe recurrir al uso de algún método o técnica para eliminar la variabilidad de fase, manteniendo la información de la función contenida en la forma y la amplitud de las señales.

La técnica estándar usada en biomecánica es la normalización lineal de la escala de tiempos, que consiste en transformar la base de tiempos en una escala de 0 a $100 \%$ del tiempo de ejecución del movimiento. Es una técnica simple y efectiva (Boulgouris et al., 2006). No obstante, otros estudios han demostrado que dicha técnica puede ser inadecuada en determinados casos. En particular, en el trabajo de Page y Epifanio (2007) se definen los criterios matemáticos que determinan la la efectividad de la normalización lineal en la reducción de la variabilidad de los datos, basándose en la correlación entre la duración total de cada ciclo y la duración de los eventos principales de la función.

Por ello se han planteado otras alternativas, como el registro de funciones. Se trata de una técnica de normalización no lineal, que expande o contrae localmente la escala de tiempos para buscar la mejor adaptación entre dos curvas, de manera que se minimice el efecto de las diferencias de fase (Ramsay \& Li, 1998). Los métodos de registro de funciones eliminan prácticamente toda la variabilidad de fase, de manera que las curvas registradas tienen la misma forma, y sólo se diferencian por las distintas amplitudes. Esto permite obtener buenas estimaciones de las curvas promedio. Sin embargo, la información temporal (que en la normalización lineal se mantiene en los desfases entre curvas), no desaparece en este tipo de reescalado, sino que se mantiene en las "time warping functions". Estas funciones representan la relación entre el tiempo modificado en función del tiempo real, $h(t)$ y sirven para analizar en qué momentos el movimiento se desarrolla más deprisa o más lento que el promedio del movimiento (Page et al, 2006; Crane et al., 2010).

A pesar de lo atractivo que resulta el registro no lineal de funciones, hay que señalar que presenta algunas limitaciones. En primer lugar, hay que destacar que su efectividad en la reducción de variabilidad depende del tipo y características de las funciones a registrar. Así, cuando se aplica a curvas de movimiento que presentan un gran número de puntos característicos (máximos, mínimos, puntos de inflexión) la reducción en la variabilidad es mucho mejor que cuando se aplica a curvas con pocos puntos característicos (Crane et al., 2010). Además, el proceso de registro es complejo desde el punto de vista matemático, y muy costoso desde el punto de vista computacional, ya que las curvas deben registrarse una a una respecto a una de referencia (típicamente una media). $\mathrm{Si}$, como es frecuente, se usa un proceso procústeo de adaptación de la media, entonces el cálculo se hace muy pesado (Page et al, 2006). Finalmente, el registro de funciones se basa en criterios meramente geométricos que no tienen en cuenta la relación dinámica entre las funciones y sus 
derivadas. Por ello, es posible que los registros obtenidos con una función o su derivada arrojen resultados distintos, lo que no tiene sentido desde el punto de vista físico

En definitiva, cabe valorar las ventajas comparativas del registro frente a la normalización no lineal y evaluar si la mejora en la obtención de curvas promedio compensa la complejidad o posibles patrones anómalos asociados al registro no lineal.

En esta línea se plantea el presente trabajo, cuyo objetivo es comparar ambos tipos de normalizaciones en una amplia base de curvas de flexión-extensión del cuello, analizando tanto los funciones correspondientes al ángulo como a la velocidad angular. En particular comprobaremos las diferencias a la hora de determinar las medias y desviaciones típicas funcionales y las diferencias obtenidas por el registro según se usen los ángulos o las velocidades angulares.

\section{Material y métodos}

\subsection{Muestra de estudio y funciones analizadas.}

Las funciones utilizadas en este trabajo proceden de un estudio sobre el movimiento del cuello en el que participaron 15 sujetos que fueron medidos en dos sesiones diferentes y por dos operadores distintos. Los sujetos fueron voluntarios sanos que firmaron el correspondiente consentimiento informado, de acuerdo con un protocolo aprobado por el Comité de Ética de la Universitat de València.

En cada sesión de medida el sujeto realizó un movimiento continuo de flexo-extensión, describiendo varios ciclos seguidos del movimiento sin detenerse. El protocolo está descrito en Baydal et al.(2011).

\subsection{Captura de movimientos y procesado de las señales.}

El movimiento fue medido con un equipo de fotogrametría (Kinescan-IBV) a 200 fotogramas por segundo. El sistema proporciona las coordenadas de los marcadores. A partir de dichas coordenadas se utilizó software propio para la medida del ángulo de flexoextensión y su derivada (Baydal. 2011). Posteriormente, el movimiento continuo fue dividido en ciclos, cada uno de los cuales es un observación de la muestra. En total se obtuvieron 437 ciclos completos. 


\subsection{Normalizacion lineal y registos}

Sobre la base de 437 ciclos se efectuaron dos normalizaciones:normalización lineal y registro de funciones.

\subsubsection{Normalización lineal}

Se normalizaron las medidas de velocidades cambiando la base de tiempos por un procentaje de la duración de cada ciclo, de manera que el tiempo en el instante i, de la curva $\mathrm{j}, \mathrm{t}_{\mathrm{ij}}$, se transforma en $\mathrm{p}_{\mathrm{ij}}$ :

$$
p_{i j}=100 \times \frac{t_{i j}}{T_{j}}
$$

Donde $\mathrm{T}_{\mathrm{j}}$ es la duración de la curva $\mathrm{j}$. Las curvas normalizadas se interpolaron para obtener un conjunto de 101 valores, desde $\mathrm{p}=0$ hasta $\mathrm{p}=100$.

\subsubsection{Registro de funciones}

Para la normalización no lineal se usó el procedimiento descrito en Page et al. (2006). Con el registro de funciones se pretende encontrar una transformación de la escala de tiempos, $\mathrm{h}_{\mathrm{i}}$, que permite deformar la curva inicial para minimizar las diferencias de fase:

$$
x_{i}^{*}(t)=x_{i}\left(h_{i}(t)\right)
$$

La función $h_{\mathrm{i}}$ se denomina warping function, y debe ser una función continua, derivable y monótona creciente. Estas funciones representan una transformación no lineal de la escala de tiempos, de manera que el tiempo se contrae o dilata localmente para ajustar la forma de las curvas. Por tanto, contienen la información sobre las diferencias temporales que se pierde cuando se alinean las curvas de forma continua al registrarlas. En definitiva, representan la variabilidad en la fase, mientras que las curvas registradas contienen la variabilidad asociada a las diferencias de amplitud.

El criterio usado para minimizar las diferencias de fase consiste en mimizar la función $\mathrm{F}$ (Page et al., 2006)

$$
F\left(h_{i}\right)=\log \mu_{2}(\Sigma)
$$

Donde $\mu_{2}$ es el menor valor propio de la matriz $\Sigma$, que corresponde con una matriz de varianzas-covarianzas de las curvas que se van a registrar:

$$
\Sigma=\left(\begin{array}{cc}
\int x_{0}^{2}(t) d t & \int x_{0}(t) x_{i}(t) d t \\
\int x_{0}(t) x_{i}(t) d t & \int x_{i}^{2}(t) d t
\end{array}\right)
$$


El proceso se registro se realizó utilizando las curvas de velocidad, ya que se recomienda usar derivadas en vez de las funciones medidas (Ramsay y Silverman, 2005).

\subsubsection{Análisis estadístico}

Para evaluar las diferencias entre los tres conjuntos de funciones (brutas, normalizadas linealmente y registradas) se han realizado los siguientes análisis:

1) Análisis descriptivo, representando las familias de curvas y sus medias. Se ha realizado tanto para las curvas de posición como para las de velocidad.

2) Comparación entre los dos métodos de normalización. Para cada familia de curvas (ángulo y velocidad angular) se han calculado dos variables que cuantifican el nivel de alineamiento entre las curvas tras la normalización:

a. Rango de la curva media. Se asume que las curvas con más diferencia de fase presentan un efecto de compensación en los máximos y mínimos, de manera que la curva media tiene un rango menor.

b. Valor máximo de la desviación típica funcional. Las curvas desfasadas presentan valores de desviación típica muy grandes en los puntos de inflexión. Se asume que el método que mejor ajuste los desfases, tendrá menores valores máximos de la desviación típica

Se ha elaborado un contraste estadístico para saber si las diferencias entre los dos métodos son significativas. Para ello se ha utlizado un proceso de bootstrap (con extracción de 5000 muestras con reemplazo) obteniedo en intervalo de confianza de la diferencia. Ajustando el valor de alfa para que el límie inferior sea muy próximo a cero se obtiene en nivel de significación (Efron, 1994).

\section{Resultados}

La Figura 1 muestra la distribución de la familia de curvas con la escala de tiempos sin normalizar. Como puede observarse, las curvas presentan una gran dispersión en amplitud y en la duración de los ciclos.

Como consecuencia de estas diferencias, los valores medios están algo truncados en los valores extremos. Además la curva media se vuelve inestables y su forma está toalmente alterada al final del movimiento. 

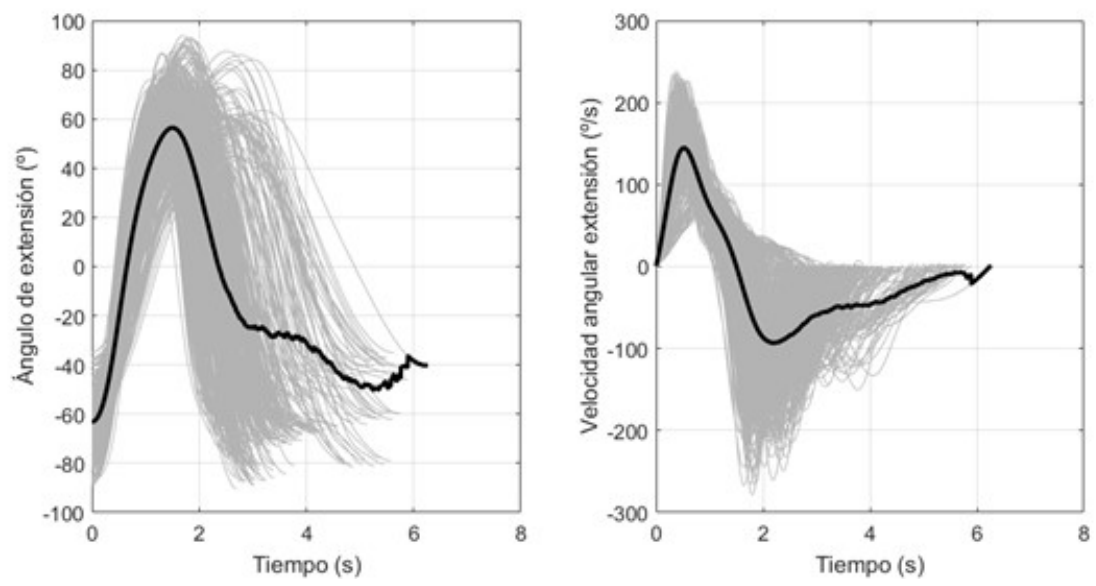

Fig. 1. Ángulo y velocidad angular con la base de tiempos bruta. En gris las 437 curvas usadas. La línea negra representa la media.

Las figuras 2 y 3 muestran la misma representación, pero con la normalización lineal (Fig. 2) y tras el registro de funciones (Fig.3). La normalización lineal proporciona curvas suaves. No obstante, se mantienen ciertas diferencias de fase, lo que se puede traducir en una disminución del valor máximo y mayor dispersión.
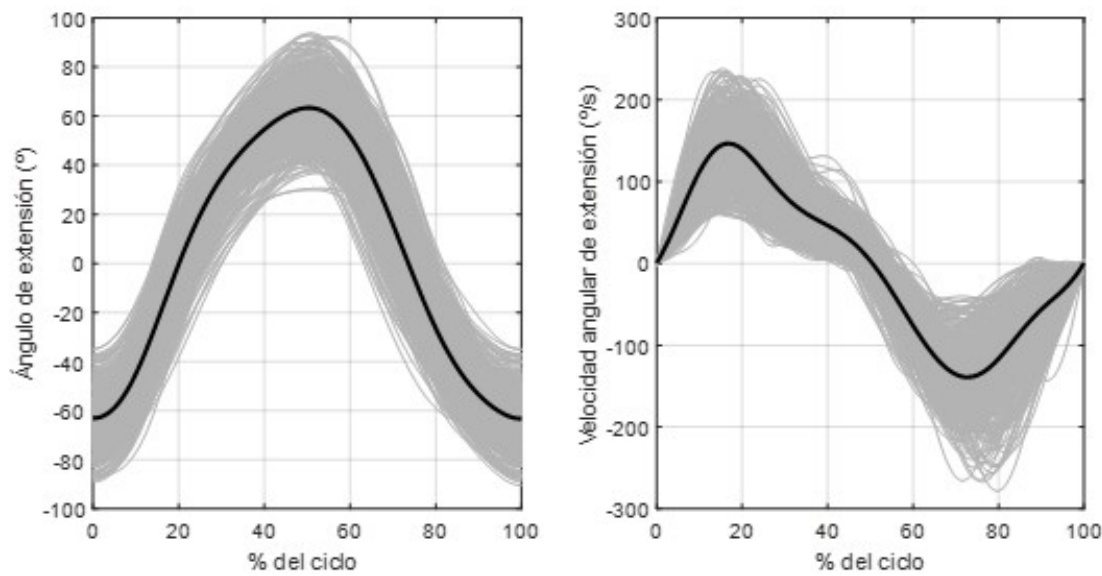

Fig. 2. Angulo y velocidad angular con la base de tiempos normalizada linealmente. En gris las 437 curvas usadas. La línea negra representa la media.

Por el contrario, las curvas registradas presentan un alineamiento mejor, especialmente en las curvas de velocidad, que han sido las usadas para obtener el registro. Sin embargo, en 
las curvas de los ángulos se aprecian ciertas deformaciones en algunas curvas que, una vez registradas no mantienen la forma original. Por este motivo la curva media tienen una pequeña deformación.
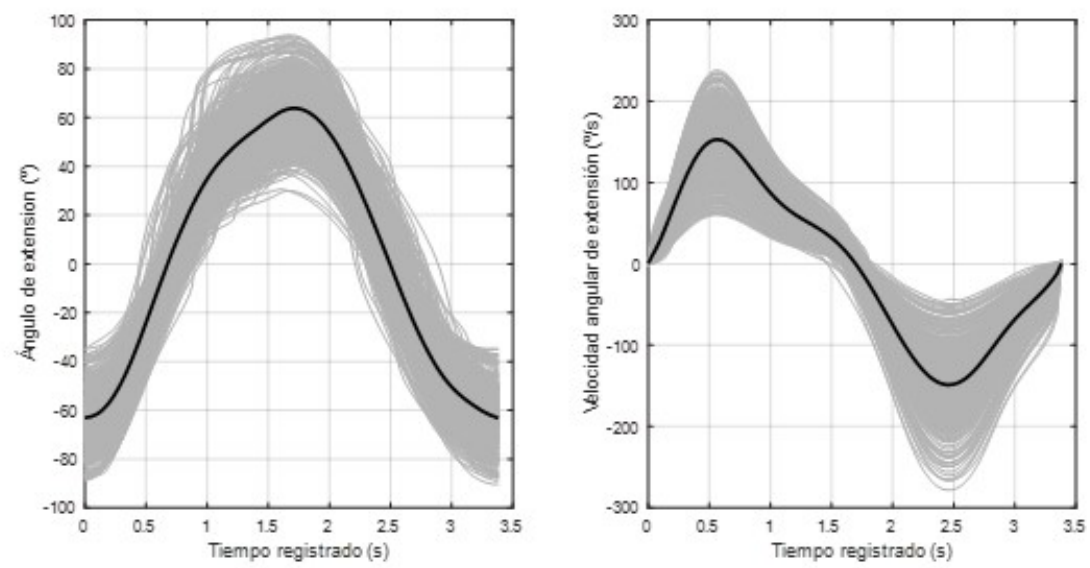

Fig. 3. Ángulo y velocidad angular con la base de tiempos normalizada mediante registro de funciones. En gris las 437 curvas usadas. La linea negra representa la media.

Tabla 1. Diferencias entre la curvas originales, la normalización lineal y la normalización no lineal. Los niveles de significación se refieren a las diferencias entre la normalización no lineal y el registro de funciones.

\begin{tabular}{ccccc} 
& $\begin{array}{c}\text { Sin } \\
\text { normalizar }\end{array}$ & $\begin{array}{c}\text { Normalización } \\
\text { lineal }\end{array}$ & $\begin{array}{c}\text { Registro no } \\
\text { lineal }\end{array}$ & $\begin{array}{c}\text { Nivel } \\
\text { significación }\end{array}$ \\
\hline $\begin{array}{c}\text { Amplitud de la curva } \\
\text { media de angulos }\left(^{(}\right)\end{array}$ & 119.5 & 126.6 & 127.1 & $<0.01$ \\
$\begin{array}{c}\text { Amplitud de la curva } \\
\text { media de velocidades }\left(\mathbf{(}^{\circ} / \mathbf{s}\right)\end{array}$ & 237.8 & 285.5 & 301.6 & $<0.0001$ \\
$\begin{array}{c}\text { Valor máximo, DT } \\
\text { funcional. Ángulo }\left({ }^{(}\right)\end{array}$ & 44.0 & 15.7 & 13.4 & $<0.0001$ \\
$\quad \begin{array}{c}\text { Valor máximo. DT } \\
\text { funcional. Velocidades }(\mathbf{\%} / \mathbf{s})\end{array}$ & 76.2 & 47.2 & 42.7 & $<0.0001$ \\
\hline
\end{tabular}

Las diferencias entre métodos de normalización se resumen en la Tabla 1. Como puede observarse, ambos métodos de normalización mejoran notablemente los parámetros 
obtenidos para las curvas brutas: tienen un rango mucho mayor y hay una disminución drástica de la desviación típica funcional.

En cuanto a las diferencias entre métodos de normalización, el registro no lineal proporciona medias con un rango significativamente mayor al de la normalización lineal , y alinea las curvas de manera que la desviación típica máxima es inferior. No obstante, las diferencias son relativamente pequeñas frente al rango. Por otra parte, también hay una disminución notable en las máximas desviaciones típicas de las curvas brutas y tras la normalización, tanto si se hace linealmente como con registro de funciones. Las diferencias entre métodos son mucho más pequeñas; si bien el registro proporciona curvas con desviaciones típicas significativamente inferiores, la tales diferencias son pequeñas si se comparan con el rango de los ángulos o las velocidades.

\section{Discusión}

La normalización de la escala de tiempos es un paso necesario para comparar las magnitudes asociadas al movimiento humano. Tradicionalmente, el problema de las diferencias en la duración y fases de las curvas asociadas al movimiento se ha resuelto mediante una normalización lineal. Esto puede dar lugar a problemas difíciles de resolver en algunos casos, como se discute en Page et al. (2006) en un estudio sobre fuerzas al sentarse y levantarse. Por este motivo, se han planteado otros procedimientos más complejos para un ajuste no lineal del tiempo, como el método del registro de funciones. Esta técnica se ha usado con éxito en muchas aplicaciones (Page et al, 2006; Sørensen \& Sangalli, 2013).

A pesar de las ventajas que puede puede presentar, esta técnica no está exenta de problemas como los señalados en Crane et al. (2010), quien sugiere que el registro de funciones no difiere de la normalización lineal en movimientos armónicos. Esta hipótesis es compatible con los criterios señalados en Page \& Epifanio (2007): cuando la duración de cada evento es proporcional a la duración del ciclo completo, como sucede en un movimiento armónico, entonces el ajuste lineal es totalmente efectivo.

Sin embargo, no se ha analizado el comportamiento los diferentes métodos de normalización en movimientos cíclicos, pero no armónicos, como es el caso del movimiento cíclico de flexo-extensión del cuello. Este es un movimiento con una armonía relativamente baja (Baydal et al., 2011) ya que no se trata de un movimiento puramente mecánico, como sucede en otros, como la elevación del brazo por ejemplo (Cáceres, 2019).

Los resultados obtenidos muestran que tanto la normalización lineal como el registro de funciones proporcionan una importante reducción de la variabilidad asociada a las 
diferencias de fase. La alineación de las curvas es mejor en el caso del registro, si bien hay algunos matices.

Respecto a las curvas de flexo-extensión, el método del registro produce deformación local en algunas curvas, debidos a cambios acusados en las funciones warping. Sin embargo, la media tiene una amplitud ligeramente superior a la obtenida con la simple normalización lineal. Aunque las diferencias son significativas, son realmente pequeñas si se comparan con el rango (menos del $0.5 \%$ ). Lo mismo sucede con la máxima desviación típica que presenta pequeñas diferencias entre los dos métodos. Teniendo en cuenta que el registro altera de forma anormal la forma de algunas curvas, cabe plantearse si en este caso tiene más ventajas o inconvenientes que el método más simple.

En cuanto a las curvas de velocidad, aquí las diferencias son algo más acusadas. Las curvas de velocidad registradas son suaves y mantienen formas muy similares, sin deformaciones. Proporcionan una media algo mayor que la obtenida tras la normalización lineal y también reduce algo más la variabilidad, como se ve en las desviaciones típicas. Por ello, parece que la normalización no lineal es algo más efectiva que la lineal en las curvas de velocidad

Estas diferencias entre la efectividad en las funciones de posición y de velocidad pueden deberse, en nuestra opinión, a dos factores. Por una parte a que el registro se ha hecho tomando las velocidades como función a registrar, aplicando posteriormente las funciones warping a las posiciones. Por otra, debido a que las curvas de velocidad se parecen menos a una función armónica que las de posición.

En cualquier caso las diferencias son pequeñas y la complejidad matemática y coste computacional del registro de funciones puede no compensar el uso de esta técnica en movimientos cíclicos.

\section{Conclusiones}

A pesar de su sencillez la normalización lineal de la escala de tiempos es una técnica efectiva para reducir la variabilidad entre curvas asociadas a las diferencias de fase en el caso de movimientos cíclicos.

Otras alternativas más complejas, como el registro de funciones reduce algo más la variabilidad, pero la mejora no compensa la complejidad del método. Además, al registrar las derivadas las funciones primitivas pueden alterarse, lo que implica que no se respeta la dinámica del movimiento. Quizás la utilidad de estas técnicas sea mayor en el caso de funciones claramente no armónicas donde no interesan las derivadas, como en el caso de los registros de fuerzas. Sin embargo, cuando deban analizarse a la vez funciones y sus 
derivadas (como en los estudios de coordinación) deberían utilizarse la normalización lineal o buscar nuevas alternativas si dicha normalización no ofrece resultados satrisfactorios.

\section{Agradecimientos.}

Este trabajo ha sido financiado por el Gobierno de España y cofinanciado por Fondos Feder de la UE gracias al proyecto PDI2017-84201-R (IMBIO3R).

\section{Bibliografía}

BAYDAL-BERTOMEU, J. M., PAGE, Á. F., BELDA-LOIS, et al. (2011). "Neck motion patterns in whiplash-associated disorders: quantifying variability and spontaneity of movement". Clinical Biomechanics, vol. 26, issue 1, pp. 29-34.

BOUlgOURIS, N. V., PLATANIOTIS, K. N., \& HATZINAKOS, D. (2006). "Gait recognition using linear time normalization”. Pattern Recognition, vol. 39, issue 5, pp. 969-979.

CÁCERES, M.L. (2019). "Aplicación de la cinemática articular a la valoración funcional del hombro". Tesis Doctoral. Universitat Politécnica de Valencia.

CRANE, E. A., et al. (2010). "Effect of registration on cyclical kinematic data". Journal of biomechanics, vol 43, issue 12, pp 2444-2447.

CRANE, E., CHILDERS, D., GERSTNER, G., \& ROTHMAN, E. (2011). "Functional data analysis for biomechanics". In: Theoretical Biomechanics. IntechOpen.

EFRON, B., \& TIBSHIRANI, R. J. (1994). An introduction to the bootstrap. CRC press.

LIEBL, D., MOSLER, K., \& WILLWACHER, S. (2012). "Robust clustering of joint moment curves". Sportinformatik, pp 68.

LUCERO, J. C., \& KOENIG, L. L. (2000). "Time normalization of voice signals using functional data analysis". The Journal of the Acoustical Society of America, vol. 108, issue 4, pp. 1408-1420.

MECHMECHE, I., MITICHE, A., OUAKRIM, Y., et al. (2016). "Data correction to determine a representative pattern of a set of 3D knee kinematic measurements". In: 2016 38th Annual International Conference of the IEEE Engineering in Medicine and Biology Society (EMBC), pp. 884-887). IEEE.

NOVACHECK, T. F. (1998). "The biomechanics of running”. Gait \& posture, vol 7, issue 1, pp. 7795.

PAGE, A., \& EPIFANIO, I. (2007). "A simple model to analyze the effectiveness of linear time normalization to reduce variability in human movement analysis". Gait \& posture, vol. 25, issue1, pp. 153-156. 
Comparación de la normalización lineal de la escala de tiempos con el registro funcional continuo en movimientos cíclicos del cuello

PAGE, A., AYALA, G., LEON, M. T.,et al. (2006). "Normalizing temporal patterns to analyze sit-tostand movements by using registration of functional data". Journal of biomechanics, vol. 39, issue 13, pp. 2526-2534.

RAMSAY, J. O., \& LI, X. (1998). "Curve registration". Journal of the Royal Statistical Society: Series B (Statistical Methodology), vol 60, issue 2, pp. 351-363.

RAMSAY, J. O., \& SILVERMAN, B. W. (2007). "Applied functional data analysis: methods and case studies". Springer.

SNOOK, S. H., \& CIRIELLO, V. M. (1991). "The design of manual handling tasks: revised tables of maximum acceptable weights and forces”. Ergonomics, vol. 34, issue 9, pp. 1197-1213.

SØRENSEN, H., GOLDSMITH, J., \& SANGALLI, L. M. (2013). "An introduction with medical applications to functional data analysis". Statistics in Medicine, vol 32 issue 30, pp. 5222-5240.

ULLAH, S., \& FINCH, C. F. (2013). "Applications of functional data analysis: A systematic review". BMC Medical Research Methodology, 13(1), 43.

WINTER; D.A. (1991). "The biomechanics and motor control of human gait : normal, elderly and pathological”. University of Waterloo Press. Waterloo. 